Pacific

Journal of

Mathematics

\title{
A VARIATIONAL CHARACTERIZATION OF FLAT SPACES IN DIMENSION THREE
}

Giovanni Catino, Paolo Mastrolia and Dario D. Monticelli 


\title{
A VARIATIONAL CHARACTERIZATION OF FLAT SPACES IN DIMENSION THREE
}

\author{
Giovanni Catino, Paolo Mastrolia and Dario D. Monticelli
}

We prove that, in dimension three, flat metrics are the only complete metrics with nonnegative scalar curvature which are critical for the $\sigma_{2}$-curvature functional.

\section{Introduction}

Let $\left(M^{n}, g\right)$ be a Riemannian manifold of dimension $n \geq 3$. To fix the notation, we recall the decomposition of the Riemann curvature tensor of a metric $g$ into the Weyl, Ricci, and scalar curvature components:

$$
\mathrm{Rm}=W+\frac{1}{n-2} \operatorname{Ric} \otimes g-\frac{1}{(n-1)(n-2)} \operatorname{Rg} \otimes g,
$$

where $\otimes$ denotes the Kulkarni-Nomizu product. It is well known [Hilbert 1915] that Einstein metrics are critical points for the Einstein-Hilbert functional

$$
\mathcal{H}=\int R d V
$$

on the space of unit volume metrics $M_{1}\left(M^{n}\right)$. From this perspective, it is natural to study canonical metrics which arise as solutions of the Euler-Lagrange equations for more general curvature functionals. Berger [1970] commenced the study of Riemannian functionals which are quadratic in the curvature (see [Besse 2008, Chapter 4] and [Smolentsev 2005] for surveys). A basis for the space of quadratic curvature functionals is given by

$$
\mathcal{W}=\int|W|^{2} d V, \quad \rho=\int|\operatorname{Ric}|^{2} d V, \quad \mathcal{S}=\int R^{2} d V .
$$

All such functionals, which also naturally arise as total actions in certain gravitational field theories in physics, have been deeply studied in recent years by many authors, in particular on compact Riemannian manifolds with normalized volume (for instance, see [Berger 1970; Besse 2008; Lamontagne 1994; 1998; Anderson 1997; Gursky and Viaclovsky 2001; 2015; 2013; Catino 2015] and references therein).

Keywords: critical metrics, quadratic functionals, rigidity results. 
On the other hand, the study of critical metrics for quadratic curvature functionals also has a lot of interest in the noncompact setting. For instance, Anderson [2001] proved that every complete three-dimensional critical metric for the Ricci functional $\rho$ with nonnegative scalar curvature is flat; whereas, Catino [2014] showed a characterization of complete critical metrics for $\mathcal{S}$ with nonnegative scalar curvature in every dimension.

In this paper we focus our attention on the three-dimensional case and consider the $\sigma_{2}$-curvature functional

$$
\mathscr{F}_{2}=\int \sigma_{2}(A) d V
$$

where $\sigma_{2}(A)$ denotes the second elementary symmetric function of the eigenvalues of the Schouten tensor $A=\mathrm{Ric}-\frac{1}{4} R g$. This functional was first considered by Gursky and Viaclovsky in the compact three-dimensional case. In [2001] they proved a beautiful characterization theorem of space forms as critical metrics for $\mathscr{F}_{2}$ on $M_{1}\left(M^{3}\right)$ with nonnegative energy $\mathscr{F}_{2} \geq 0$.

The main result of this paper is the following variational characterization of three-dimensional flat spaces.

Theorem 1.1. Let $\left(M^{3}, g\right)$ be a complete critical metric for $\mathscr{F}_{2}$ with nonnegative scalar curvature. Then $\left(M^{3}, g\right)$ is flat.

We remark the fact that the nonnegativity condition on the scalar curvature cannot be removed. This is clear from the example in [loc. cit.] where the authors exhibit an explicit family of critical metrics for $\mathscr{F}_{2}$ on $\mathbb{R}^{3}$. For instance, the metric given in standard coordinates by

$$
g=d x^{2}+d y^{2}+\left(1+x^{2}+y^{2}\right)^{2} d z^{2}
$$

is complete, critical and has strictly negative scalar curvature

$$
R=-\frac{8}{1+x^{2}+y^{2}}
$$

\section{The Euler-Lagrange equation for $\mathcal{F}_{t}$}

In this section we will compute the Euler-Lagrange equation satisfied by critical metrics for $\mathscr{F}_{2}$. To begin, we observe that, in dimension $n \geq 3$, the second elementary symmetric function of the eigenvalues of the Schouten tensor

$$
A=\frac{1}{n-2}\left(\operatorname{Ric}-\frac{1}{2(n-1)} R g\right)
$$

can be written as

$$
\sigma_{2}(A)=-\frac{1}{2(n-2)^{2}}|\operatorname{Ric}|^{2}+\frac{n}{8(n-1)(n-2)^{2}} R^{2} .
$$


In particular, the functional $\mathscr{F}_{2}$ is proportional to a general quadratic functional of the form

$$
\mathcal{F}_{t}=\int|\mathrm{Ric}|^{2} d V+t \int R^{2} d V
$$

with the choice $t=-n / 4(n-1)$; see also [Gursky and Viaclovsky 2015; Catino 2015]. The gradients of the functionals $\rho$ and $\mathcal{S}$, computed using compactly supported variations, are given by [Besse 2008, Proposition 4.66]

$$
(\nabla \rho)_{i j}=-\Delta R_{i j}-2 R_{i k j l} R_{k l}+\nabla_{i j}^{2} R-\frac{1}{2}(\Delta R) g_{i j}+\frac{1}{2}|\operatorname{Ric}|^{2} g_{i j}
$$

and

$$
(\nabla \mathcal{S})_{i j}=2 \nabla_{i j}^{2} R-2(\Delta R) g_{i j}-2 R R_{i j}+\frac{1}{2} R^{2} g_{i j} .
$$

Hence, the gradient of $\mathcal{F}_{t}$ reads

$$
\begin{aligned}
\left(\nabla \mathcal{F}_{t}\right)_{i j}=-\Delta R_{i j}+(1+2 t) \nabla_{i j}^{2} R- & \frac{1}{2}(1+4 t)(\Delta R) g_{i j} \\
& +\frac{1}{2}\left(|\mathrm{Ric}|^{2}+t R^{2}\right) g_{i j}-2 R_{i k j l} R_{k l}-2 t R R_{i j} .
\end{aligned}
$$

Tracing the equation $\left(\nabla \mathcal{F}_{t}\right)=0$, we obtain

$$
(n+4(n-1) t) \Delta R=(n-4)\left(|\operatorname{Ric}|^{2}+t R^{2}\right) .
$$

Defining the tensor $E$ to be the traceless Ricci tensor, $E_{i j}=R_{i j}-\frac{1}{n} R g_{i j}$, we obtain the Euler-Lagrange equation of critical metrics for $\mathcal{F}_{t}$.

Proposition 2.1. Let $M^{n}$ be a complete manifold of dimension $n \geq 3$. A metric $g$ is critical for $\mathcal{F}_{t}$ if and only if it satisfies

$$
\begin{aligned}
\Delta E_{i j}=(1+2 t) & \nabla_{i j}^{2} R-\frac{n+2+4 n t}{2 n}(\Delta R) g_{i j} \\
& -2 R_{i k j l} E_{k l}-\frac{2+2 n t}{n} R E_{i j}+\frac{1}{2}\left(|\mathrm{Ric}|^{2}-\frac{4-n(n-4) t}{n^{2}} R^{2}\right) g_{i j}
\end{aligned}
$$

and

$$
(n+4(n-1) t) \Delta R=(n-4)\left(|\mathrm{Ric}|^{2}+t R^{2}\right) .
$$

In dimension three we recall the decomposition of the Riemann curvature tensor

$$
R_{i k j l}=E_{i j} g_{k l}-E_{i l} g_{j k}+E_{k l} g_{i j}-E_{k j} g_{i l}+\frac{1}{6} R\left(g_{i j} g_{k l}-g_{i l} g_{j k}\right) .
$$

In particular,

$$
R_{i k j l} E_{k l}=-2 E_{i p} E_{j p}-\frac{1}{6} R E_{i j}+|E|^{2} g_{i j} .
$$

Hence, if $n=3$ and $t=-n / 4(n-1)=-3 / 8$, one has

$$
\mathscr{F}_{2}=-\frac{1}{2} \mathcal{F}_{-3 / 8},
$$

and the following formulas hold. 
Proposition 2.2. Let $M^{3}$ be a complete manifold of dimension three. A metric $g$ is critical for $\mathscr{F}_{2}$ if and only if it satisfies

$$
\Delta E_{i j}=\frac{1}{4} \nabla_{i j}^{2} R-\frac{1}{12}(\Delta R) g_{i j}+4 E_{i p} E_{j p}+\frac{5}{12} R E_{i j}-\frac{1}{2}\left(3|E|^{2}-\frac{1}{72} R^{2}\right) g_{i j}
$$

and

$$
-2 \sigma_{2}(A)=|\mathrm{Ric}|^{2}-\frac{3}{8} R^{2}=|E|^{2}-\frac{1}{24} R^{2}=0 .
$$

Now, contracting (2-1) with $E$, we obtain the following Weitzenböck formula.

Corollary 2.3. Let $M^{3}$ be a complete manifold of dimension three. If $g$ is a critical metric for $\mathscr{F}_{2}$, then the following formula holds

$$
\frac{1}{2} \Delta|E|^{2}=|\nabla E|^{2}+\frac{1}{4} E_{i j} \nabla_{i j}^{2} R+4 E_{i p} E_{j p} E_{i j}+\frac{5}{12} R|E|^{2} .
$$

\section{Proof of Theorem 1.1}

In this section we will prove Theorem 1.1. We assume that $\left(M^{3}, g\right)$ is a critical metric for $\mathscr{F}_{2}$ with nonnegative scalar curvature $R \geq 0$. In particular, $g$ has zero $\sigma_{2}$-curvature, i.e., $|E|^{2}=\frac{1}{24} R^{2}$ and we obtain

$$
\frac{1}{2} \Delta|E|^{2}=\frac{1}{48} \Delta R^{2}=\frac{1}{24} R \Delta R+\frac{1}{24}|\nabla R|^{2} .
$$

Putting together this equation with (2-3), we obtain that the scalar curvature $R$ satisfies the PDE

$$
\frac{1}{24}\left(R g_{i j}-6 E_{i j}\right) \nabla_{i j}^{2} R=|\nabla E|^{2}-\frac{1}{24}|\nabla R|^{2}+4 E_{i p} E_{j p} E_{i j}+\frac{5}{12} R|E|^{2} .
$$

To begin, we need the following purely algebraic lemmas.

Lemma 3.1. Let $\left(M^{3}, g\right)$ be a Riemannian manifold with $R \geq 0$ and $\sigma_{2}(A) \geq 0$. Then,

$$
R g_{i j} \geq 6 E_{i j}
$$

and $g$ has nonnegative sectional curvature.

Proof. Let $\lambda_{1} \leq \lambda_{2} \leq \lambda_{3}$ be the eigenvalues of the Schouten tensor $A=E+\frac{1}{12} R g$ at some point. Then, by the assumptions, we have

$$
4 R=\operatorname{tr}(A)=\lambda_{1}+\lambda_{2}+\lambda_{3} \geq 0 \quad \text { and } \quad \sigma_{2}(A)=\lambda_{1} \lambda_{2}+\lambda_{1} \lambda_{3}+\lambda_{2} \lambda_{3} \geq 0 .
$$

We want to show that $E \leq \frac{1}{6} R g$ or, equivalently, that

$$
A \leq \frac{1}{4} R g=\operatorname{tr}(A) g .
$$

Hence, it suffices to prove that $\lambda_{3} \leq \operatorname{tr}(A)=\lambda_{1}+\lambda_{2}+\lambda_{3}$, i.e., that $\lambda_{1}+\lambda_{2} \geq 0$. But this follows by

$$
0 \leq \lambda_{1} \lambda_{2}+\lambda_{1} \lambda_{3}+\lambda_{2} \lambda_{3}=\left(\lambda_{1}+\lambda_{2}\right) \operatorname{tr}(A)-\left(\lambda_{1}^{2}+\lambda_{2}^{2}+\lambda_{1} \lambda_{2}\right) \leq\left(\lambda_{1}+\lambda_{2}\right) \operatorname{tr}(A) .
$$


The fact that $g$ has nonnegative sectional curvature follows from the decomposition of the Riemann tensor in dimension three and the curvature condition Ric $\leq \frac{1}{2} R g$ (for instance see [Hamilton 1982, Corollary 8.2]).

Lemma 3.2. Let $\left(M^{3}, g\right)$ be a Riemannian manifold with $R \geq 0$ and $\sigma_{2}(A)=$ const $\geq 0$. Then,

$$
|\nabla E|^{2} \geq \frac{1}{24}|\nabla R|^{2}
$$

Proof. We will follow the proof in [Gursky and Viaclovsky 2001, Lemma 4.1]. Let $p$ be a point in $M^{3}$. If $R(p)=0$, then $\nabla R=0$ and the lemma follows. So we can assume that $R(p)>0$. Since $-2 \sigma_{2}(A)=|E|^{2}-\frac{1}{24} R^{2}=$ const,

$$
|E|^{2}|\nabla| E||^{2}=\frac{1}{576} R^{2}|\nabla R|^{2} .
$$

By Kato's inequality $|\nabla| E||^{2} \leq|\nabla E|^{2}$ and the fact that $|E|^{2} \leq \frac{1}{24} R^{2}$,

$$
|E|^{2}|\nabla E|^{2} \geq \frac{1}{576} R^{2}|\nabla R|^{2} \geq \frac{1}{24}|E|^{2}|\nabla R|^{2} .
$$

By dividing by $|E|^{2}(p) \neq 0$, the result follows; otherwise, if $|E|(p)=0$, then $(\nabla R)(p)=0$ from (3-2), and we conclude.

Lemma 3.3. Let $\left(M^{3}, g\right)$ be a Riemannian manifold. Then,

$$
E_{i p} E_{j p} E_{i j} \geq-\frac{1}{\sqrt{6}}|E|^{3} .
$$

Proof. For a proof of this lemma, for instance, see [op. cit., Lemma 4.2].

Corollary 3.4. Let $\left(M^{3}, g\right)$ be a complete critical metric for $\mathscr{F}_{2}$ with nonnegative scalar curvature. Then, $R g_{i j} \geq 6 E_{i j}, g$ has nonnegative sectional curvature, and the scalar curvature satisfies the differential inequality

$$
\left(R g_{i j}-6 E_{i j}\right) \nabla_{i j}^{2} R \geq \frac{1}{12} R^{3} .
$$

Proof. From (3-1), combining Lemmas 3.1, 3.2, and 3.3, we obtain

$$
\frac{1}{24}\left(R g_{i j}-6 E_{i j}\right) \nabla_{i j}^{2} R \geq \frac{5}{12} R|E|^{2}-\frac{4}{\sqrt{6}}|E|^{3}=|E|^{2}\left(\frac{5}{12} R-\frac{4}{\sqrt{6}}|E|\right)=\frac{1}{288} R^{3},
$$

where in the last equality we have used the fact that $|E|^{2}=\frac{1}{24} R^{2}$.

Now we can prove Theorem 1.1. Clearly, if $M^{3}$ is compact, from Corollary 3.4, at a maximum point of $R$ we obtain $R \leq 0$. Hence, $R \equiv 0$ on $M^{3}$, and from (2-2), Ric $\equiv 0$ and the metric is flat. So, from now on, we will assume the manifold $M^{3}$ to be noncompact.

Choose now $\phi=\phi(r)$ to be a function of the distance $r$ to a fixed point $O \in M^{3}$ and let $B_{s}(O)$ be a geodesic ball of radius $s>0$. We denote by $C_{O}$ the cut locus at 
the point $O$ and we choose $\phi$ satisfying the following properties: $\phi=1$ on $B_{S}(O)$, $\phi=0$ on $M^{3} \backslash B_{2 s}(O)$,

$$
-\frac{c}{s} \phi^{3 / 4} \leq \phi^{\prime} \leq 0 \quad \text { and } \quad\left|\phi^{\prime \prime}\right| \leq \frac{c}{s^{2}} \phi^{1 / 2}
$$

on $B_{2 s}(O) \backslash B_{s}(O)$ for some positive constant $c>0$. In particular, $\phi$ is $C^{3}$ in $M^{3} \backslash C_{O}$. Let $u:=R \phi$ and $a_{i j}:=\left(R g_{i j}-6 E_{i j}\right)$. From Corollary 3.4, we know that $a_{i j} \geq 0$ and we obtain

$$
\begin{aligned}
a_{i j} \nabla_{i j}^{2} u & =a_{i j}\left(\phi \nabla_{i j}^{2} R+R \nabla_{i j}^{2} \phi+2 \nabla_{i} R \nabla_{j} \phi\right) \\
& \geq \frac{1}{12} R^{3} \phi+R \phi^{\prime} a_{i j} \nabla_{i j}^{2} r+R \phi^{\prime \prime} a(\nabla r, \nabla r)+2 a(\nabla R, \nabla \phi) .
\end{aligned}
$$

Now, let $p_{0}$ be a maximum point of $u$ and assume that $p_{0} \notin C_{O}$. If $\phi\left(p_{0}\right)=0$, then $u \equiv 0$ and then $R \equiv 0$ on $B_{2 s}(O)$. Hence, from now on we will assume $\phi\left(p_{0}\right)>0$. Then, at $p_{0}$, we have $\nabla u\left(p_{0}\right)=0$ and $\nabla_{i j}^{2} u\left(p_{0}\right) \leq 0$. In particular, at $p_{0}$,

$$
\nabla R\left(p_{0}\right)=-\frac{R\left(p_{0}\right)}{\phi\left(p_{0}\right)} \nabla \phi\left(p_{0}\right) .
$$

Moreover, since $a_{i j} \geq 0$, for every vector field $X, a(X, X) \leq \operatorname{tr}(a)|X|^{2}=3 R|X|^{2}$. On the other hand, from the standard Hessian comparison theorem, since $g$ has nonnegative sectional curvature, we know that on $M^{3} \backslash C_{O}$, one has $\nabla_{i j}^{2} r \leq \frac{1}{r} g_{i j}$. Thus, from (3-3), at $p_{0}$, we get

$$
\begin{aligned}
0 & \geq \frac{1}{12} R^{3} \phi+R \phi^{\prime} a_{i j} \nabla_{i j}^{2} r+R \phi^{\prime \prime} a(\nabla r, \nabla r)-2 \frac{R}{\phi} a(\nabla \phi, \nabla \phi) \\
& \geq \frac{1}{12} R^{3} \phi-\left(\frac{\left|\phi^{\prime}\right|}{r}+\left|\phi^{\prime \prime}\right|+2 \frac{\left(\phi^{\prime}\right)^{2}}{\phi}\right) R \operatorname{tr}(a) \\
& \geq \frac{1}{12} R^{3} \phi-3\left(\frac{\left|\phi^{\prime}\right|}{s}+\left|\phi^{\prime \prime}\right|+2 \frac{\left(\phi^{\prime}\right)^{2}}{\phi}\right) R^{2},
\end{aligned}
$$

where, in the last inequality, we have used the fact that $r \geq s$ on $B_{2 s}(O) \backslash B_{s}(O)$, i.e., where $\phi^{\prime} \neq 0$. From the assumptions on the cut-off function $\phi$, we obtain, at the maximum point $p_{0}$,

$$
0 \geq \frac{1}{12} R^{2} \phi^{1 / 2}\left(R \phi^{1 / 2}-\frac{c^{\prime}}{s^{2}}\right)
$$

for some positive constant $c^{\prime}>0$. Thus, we have proved that, if $p_{0} \notin C_{O}$, then for every $p \in B_{2 s}(O)$

$$
u(p) \leq u\left(p_{0}\right)=R\left(p_{0}\right) \phi\left(p_{0}\right) \leq \frac{c^{\prime}}{s^{2}} .
$$

If $p_{0} \in C_{O}$ we argue as follows (this trick is usually referred to Calabi). Let $\gamma:[0, L] \rightarrow M^{3}$, where $L=d\left(p_{0}, O\right)$, be a minimal geodesic joining $O$ to $p_{0}$, the 
maximum point of $u$. Let $p_{\varepsilon}=\gamma(\varepsilon)$ for some $\varepsilon>0$. Define now

$$
u_{\varepsilon}(x)=R(x) \phi\left(d\left(x, p_{\varepsilon}\right)+\varepsilon\right) .
$$

Since $d\left(x, p_{\varepsilon}\right)+\varepsilon \geq d(x, O)$ and $d\left(p_{0}, p_{\varepsilon}\right)+\varepsilon=d\left(p_{0}, O\right)$, it is easy to see that $u_{\varepsilon}\left(p_{0}\right)=u\left(p_{0}\right)$ and

$$
u_{\varepsilon}(x) \leq u(x) \text { for all } x \in M^{3},
$$

since $\phi^{\prime} \leq 0$. Hence $p_{0}$ is also a maximum point for $u_{\varepsilon}$. Moreover, $p_{0} \notin C_{p_{\varepsilon}}$, so the function $d\left(x, p_{\varepsilon}\right)$ is smooth in a neighborhood of $p_{0}$ and we can apply the maximum principle argument as before to obtain an estimate for $u_{\varepsilon}\left(p_{0}\right)$ which depends on $\varepsilon$. Taking the limit as $\varepsilon \rightarrow 0$, we obtain the desired estimate on $u$.

By letting $s \rightarrow+\infty$ we obtain $u \equiv 0$, so $R \equiv 0$. From (2-2) we have $E \equiv 0$ and so Ric $\equiv 0$ and Theorem 1.1 follows.

\section{Acknowledgments}

The authors are members of the Gruppo Nazionale per l'Analisi Matematica, la Probabilità e le loro Applicazioni (GNAMPA) of the Istituto Nazionale di Alta Matematica (INdAM). Catino is supported by the GNAMPA project "Equazioni di evoluzione geometriche e strutture di tipo Einstein". Mastrolia and Monticelli are supported by the GNAMPA project "Analisi Globale ed Operatori Degeneri". Monticelli is partially supported by FSE, Regione Lombardia.

\section{References}

[Anderson 1997] M. T. Anderson, "Extrema of curvature functionals on the space of metrics on 3-manifolds", Calc. Var. Partial Differential Equations 5:3 (1997), 199-269. MR 98a:58041 Zbl 0889.58027

[Anderson 2001] M. T. Anderson, "Extrema of curvature functionals on the space of metrics on 3-manifolds, II", Calc. Var. Partial Differential Equations 12:1 (2001), 1-58. MR 2002a:58012 Zbl 1018.53020

[Berger 1970] M. Berger, "Quelques formules de variation pour une structure Riemannienne”, Ann. Sci. École Norm. Sup. (4) 3 (1970), 285-294. MR 43 \#3969 Zbl 0204.54802

[Besse 2008] A. L. Besse, Einstein manifolds, Springer, 2008. MR 2008k:53084 Zbl 1147.53001

[Catino 2014] G. Catino, "Critical metrics of the $L^{2}$-norm of the scalar curvature", Proc. Amer. Math. Soc. 142:11 (2014), 3981-3986. MR 3251738 Zbl 1300.53044

[Catino 2015] G. Catino, "Some rigidity results on critical metrics for quadratic functionals", Calc. Var. Partial Differential Equations 54:3 (2015), 2921-2937.

[Gursky and Viaclovsky 2001] M. J. Gursky and J. A. Viaclovsky, "A new variational characterization of three-dimensional space forms", Invent. Math. 145:2 (2001), 251-278. MR 2002j:53039 Zbl 1006.58008

[Gursky and Viaclovsky 2013] M. J. Gursky and J. A. Viaclovsky, "Critical metrics on connected sums of Einstein four-manifolds”, preprint, 2013. arXiv 1303.0827 
[Gursky and Viaclovsky 2015] M. J. Gursky and J. A. Viaclovsky, "Rigidity and stability of Einstein metrics for quadratic curvature functionals", J. Reine Angew. Math. 700 (2015), 37-91. MR 3318510 Zbl 06412911

[Hamilton 1982] R. S. Hamilton, "Three-manifolds with positive Ricci curvature”, J. Differential Geom. 17:2 (1982), 255-306. MR 84a:53050 Zbl 0504.53034

[Hilbert 1915] D. Hilbert, "Die Grundlagen der Physik (erste Mitteilung)”, Nachr. Ges. Wiss. Göttingen, Math.-Phys. Kl. 1915 (1915), 395-407. JFM 45.1111.01

[Lamontagne 1994] F. Lamontagne, "Une remarque sur la norme $L^{2}$ du tenseur de courbure", C. R. Acad. Sci. Paris Sér. I Math. 319:3 (1994), 237-240. MR 95i:58048 Zbl 0806.53052

[Lamontagne 1998] F. Lamontagne, "A critical metric for the $L^{2}$-norm of the curvature tensor on $S^{3}$,, Proc. Amer. Math. Soc. 126:2 (1998), 589-593. MR 98d:58035 Zbl 0904.58010

[Smolentsev 2005] N. K. Smolentsev, "Spaces of Riemannian metrics", Sovrem. Mat. Prilozh. 31 (2005), 69-147. In Russian; translated in J. Math. Sci. (New York) 142:5 (2007), 2436-2519. MR 2010a:58015 Zbl 1130.58006

Received March 15, 2015.

Giovanni Catino

DiPARTIMENTO Di MATEMATICA

POLITECNICO Di Milano

PIAZZA LEONARDO DA VINCI 32

I-20133 Milano

ITALY

giovanni.catino@polimi.it

Paolo Mastrolia

DiparTimento Di MATEMATICA

UniVERSità DEgli STUdi di Milano

Via CESARE SALDINI 50

I-20133 MiLANO

ITALY

paolo.mastrolia@unimi.it

DARIO D. MONTICELli

DipARTIMENTO Di MATEMATICA

PoliteCnico di Milano

PiAZZA LEONARDO DA VINCI 32

I-20133 Milano

ITALY

dario.monticelli@polimi.it 


\title{
PACIFIC JOURNAL OF MATHEMATICS
}

\author{
msp.org/pjm
}

Founded in 1951 by E. F. Beckenbach (1906-1982) and F. Wolf (1904-1989)

\section{EDITORS}

Don Blasius (Managing Editor)

Department of Mathematics

University of California

Los Angeles, CA 90095-1555

blasius@math.ucla.edu

\author{
Paul Balmer \\ Department of Mathematics \\ University of California \\ Los Angeles, CA 90095-1555 \\ balmer@math.ucla.edu \\ Robert Finn \\ Department of Mathematics \\ Stanford University \\ Stanford, CA 94305-2125 \\ finn@math.stanford.edu \\ Sorin Popa \\ Department of Mathematics \\ University of California \\ Los Angeles, CA 90095-1555 \\ popa@math.ucla.edu
}

\author{
Vyjayanthi Chari \\ Department of Mathematics \\ University of California \\ Riverside, CA 92521-0135 \\ chari@math.ucr.edu \\ Kefeng Liu \\ Department of Mathematics \\ University of California \\ Los Angeles, CA 90095-1555 \\ liu@math.ucla.edu \\ Jie Qing \\ Department of Mathematics \\ University of California \\ Santa Cruz, CA 95064 \\ qing@ cats.ucsc.edu
}

\section{PRODUCTION}

Silvio Levy, Scientific Editor, production@msp.org

\section{SUPPORTING INSTITUTIONS}

ACADEMIA SINICA, TAIPEI

CALIFORNIA INST. OF TECHNOLOGY

INST. DE MATEMÁTICA PURA E APLICADA

KEIO UNIVERSITY

MATH. SCIENCES RESEARCH INSTITUTE

NEW MEXICO STATE UNIV.

OREGON STATE UNIV.

\author{
STANFORD UNIVERSITY \\ UNIV. OF BRITISH COLUMBIA \\ UNIV. OF CALIFORNIA, BERKELEY \\ UNIV. OF CALIFORNIA, DAVIS \\ UNIV. OF CALIFORNIA, LOS ANGELES \\ UNIV. OF CALIFORNIA, RIVERSIDE \\ UNIV. OF CALIFORNIA, SAN DIEGO \\ UNIV. OF CALIF., SANTA BARBARA
}

\author{
Daryl Cooper \\ Department of Mathematics \\ University of California \\ Santa Barbara, CA 93106-3080 \\ cooper@math.ucsb.edu \\ Jiang-Hua Lu \\ Department of Mathematics \\ The University of Hong Kong \\ Pokfulam Rd., Hong Kong \\ jhlu@maths.hku.hk \\ Paul Yang \\ Department of Mathematics \\ Princeton University \\ Princeton NJ 08544-1000 \\ yang@math.princeton.edu
}

These supporting institutions contribute to the cost of publication of this Journal, but they are not owners or publishers and have no responsibility for its contents or policies.

See inside back cover or msp.org/pjm for submission instructions.

The subscription price for 2016 is US $\$ 440 /$ year for the electronic version, and $\$ 600 /$ year for print and electronic.

Subscriptions, requests for back issues and changes of subscriber address should be sent to Pacific Journal of Mathematics, P.O. Box 4163, Berkeley, CA 94704-0163, U.S.A. The Pacific Journal of Mathematics is indexed by Mathematical Reviews, Zentralblatt MATH, PASCAL CNRS Index, Referativnyi Zhurnal, Current Mathematical Publications and Web of Knowledge (Science Citation Index).

The Pacific Journal of Mathematics (ISSN 0030-8730) at the University of California, c/o Department of Mathematics, 798 Evans Hall \#3840, Berkeley, CA 94720-3840, is published twelve times a year. Periodical rate postage paid at Berkeley, CA 94704, and additional mailing offices. POSTMASTER: send address changes to Pacific Journal of Mathematics, P.O. Box 4163, Berkeley, CA 94704-0163.

PJM peer review and production are managed by EditFLOW ${ }^{\circledR}$ from Mathematical Sciences Publishers.

\section{PUBLISHED BY}

\section{mathematical sciences publishers \\ nonprofit scientific publishing}

http://msp.org/

(C) 2016 Mathematical Sciences Publishers 


\section{PACIFIC JOURNAL OF MATHEMATICS}

Volume $282 \quad$ No. $2 \quad$ June 2016

Exhausting curve complexes by finite rigid sets

JAVIER ARAMAYONA and CHRISTOPHER J. LEININGER

A variational characterization of flat spaces in dimension three

Giovanni Catino, Paolo Mastrolia and Dario D. Monticelli

Estimates of the gaps between consecutive eigenvalues of Laplacian

293

DAgUang Chen, TAO Zheng and HongCANG YANG

Liouville type theorems for the $p$-harmonic functions on certain manifolds

JINGYI CHEN and YUE WANG

Cartan-Fubini type rigidity of double covering morphisms of quadratic manifolds

\section{HOSUNG KIM}

On the uniform squeezing property of bounded convex domains in $\mathbb{C}^{n}$

KANG-TAE KIM and LIYOU ZHANG

Lefschetz pencils and finitely presented groups

RYOMA KOBAYASHI and NAOYUKI MONDEN

Knot homotopy in subspaces of the 3-sphere

YUYA KODA and MAKOTO OzAWA

On the relationship of continuity and boundary regularity in prescribed mean curvature Dirichlet problems

KIRK E. LANCASTER and JARON MELIN

Bridge spheres for the unknot are topologically minimal

JUNG HOON LEE

On the geometric construction of cohomology classes for cocompact discrete subgroups of $\mathrm{SL}_{n}(\mathbb{R})$ and $\mathrm{SL}_{n}(\mathbb{C})$

SUSANNE SCHIMPF

On Blaschke's conjecture

Xiaole Su, Hongwei Sun and Yusheng Wang

The role of the Jacobi identity in solving the Maurer-Cartan structure equation 